\title{
Health system factors influencing uptake of Human Papilloma Virus (HPV) vaccine among adolescent girls 9-15 years in Mbale District, Uganda
}

Juliet Nabirye ${ }^{1 *}$, Livex Andrew Okwi², Rebecca Nuwematsiko ${ }^{3}$, George Kiwanuka ${ }^{1}$, Fiston Muneza ${ }^{4}$, Carol Kamya ${ }^{1}$ and Juliet N. Babirye ${ }^{3}$

\begin{abstract}
Background: Globally, cervical cancer is the fourth most common cancer in women with more than $85 \%$ of the burden in developing countries. In Uganda, cervical cancer has shown an increase of 1.8\% per annum over the last 20 years. The availability of the Human Papillomavirus (HPV) vaccine presents an opportunity to prevent cervical cancer. Understanding how the health system influences uptake of the vaccine is critical to improve it. This study aimed to assess how the health systems is influencing uptake of HPV vaccine so as to inform policy for vaccine implementation and uptake in Mbale district, Eastern Uganda.

Methods: We conducted a cross sectional study of 407 respondents, selected from 56 villages. Six key informant interviews were conducted with District Health Officials involved in implementation of the HPV vaccine. Quantitative data was analyzed using Stata V.13. Prevalence ratios with their confidence intervals were reported. Qualitative data was audio recorded, transcribed verbatim and analyzed using MAXQDA V.12, using the six steps of thematic analysis developed by Braun and Clarke.

Results: Fifty six (14\%) of 407 adolescents self-reported vaccine uptake. 182 (52.3\%) of 348 reported lack of awareness about the HPV vaccine as the major reason for not having received it. Receiving vaccines from outreach clinics $(p=0.02)$, having many options from which to receive the vaccine $(p=0.02)$, getting an explanation on possible side-effects $(p=0.024)$, and receiving the vaccine alongside other services $(p=0.024)$ were positively associated with uptake.

Key informants reported inconsistency in vaccine supply, inadequate training on HPV vaccine, and the lack of a clear target for HPV vaccine coverage as the factors that contribute to low uptake.

Conclusion: We recommend training of health workers to provide adequate information on HPV vaccine, raising awareness of the vaccine in markets, schools, and radio talk shows, and communicating the target to health workers.

Uptake of the HPV vaccine was lower than the Ministry of Health target of $80 \%$. We recommend training of health workers to clearly provide adequate information on HPV vaccine, increasing awareness about the vaccine to the adolescents and increasing access for girls in and out of school.
\end{abstract}

Keywords: Uptake, Health system, Human papillomavirus vaccine, Cervical cancer, Low income country, Adolescent girls

\footnotetext{
* Correspondence: jbamulangeyo@gmail.com

${ }^{1}$ Department of Health Policy, Planning and Management Makerere

University School of Public Health College of Health Sciences, P.O. Box 7072,

Kampala, Uganda

Full list of author information is available at the end of the article
} 


\section{Background}

Globally, cervical cancer is the fourth most common cancer in women with more than $85 \%$ of the burden in developing countries [1]. The majority of cervical cancer mortality occurs in developing countries, where screening and optimal treatment are not adequately available [2]. Cancer of the cervix constituted $22.2 \%$ of all cancers among women in Sub-Saharan Africa in 2012 [3]. In Uganda, cervical cancer is the number one cancer killer disease among women, this is followed by breast cancer [4]. With the incidence standing at 52/100,000 women of reproductive age, it is one of the highest globally. Regrettably, more than half of these women die every year $[5,6]$. The Kampala cancer registry shows that Uganda has an age standardized incidence rate of 47.5 per 100,000 against the global estimate of 15.8 per 100,000 [7]. Many of the Cervical cancer cases present with an advanced stage of the disease [8].

Providing the Human Papilloma Virus (HPV) vaccine is aimed at primary prevention against cervical cancer so that there is no risk of infection progressing to cervical cancer later in life, because HPV is responsible for almost $90 \%$ of cervical cancer cases [9]. It is estimated that the HPV vaccine will reduce deaths from cervical cancer by two-thirds if uptake reaches $80 \%$ [10]. Two vaccines to prevent HPV infection, the cause of cervical cancer, are now approved for use in over 120 countries. This has created an opportunity to greatly enhance prevention of cervical cancer. The HPV immunization program is expected to have a significant impact on public health, however, challenges exist with delivery of the vaccine to adolescents aged 9 to 15 years which is the recommended population for HPV vaccinations by the World Health Organization [11]. This is because routine immunizations in most national programs target children younger than 5 years of age $[12,13]$. The Ministry of Health Uganda in partnership with a drug manufacturing company in the United States of America (Merk Sharpe and Dohme) launched a vaccine program in 2012, targeting 140,000 pre-adolescents. The vaccine is relatively new in Uganda, it is given out free of charge but uptake has remained low in many districts with national average estimated at $17 \%$ as of December 2016 [14]. Delivering HPV vaccines to young adolescents therefore requires a different kind of health programming [15]. Since this is a new vaccine, very few studies have been conducted about factors influencing uptake of the vaccines, therefore this study aimed to assess how the health system is influencing uptake of the HPV vaccine for adolescents 9-15 years so as to inform HPV vaccination policy and implementation program in Uganda.

\section{Methods}

\section{Study design and population}

We conducted a cross-sectional study in Mbale district in Eastern Uganda. Mbale district has a population of 488,990 people of which $52.3 \%$ are female, and $21 \%$ are between 10 and 17 years of age. The district was among the first districts where the HPV vaccination program was first implemented in 2012. We used a structured questionnaire to interview the adolescent girls. we held six key informant interviews with health workers in the district. The quantitative and qualitative data collection methods helped to obtain convergence and substantiation among the different health system factors. The multiple perspectives aimed to provide an opportunity to develop a more complete understanding of the health system factors influencing HPV vaccine uptake.

\section{Study population}

The study enrolled female adolescents aged 9-15 years because they were expected to be in Primary four or within the expected age group for the vaccination schedule.

\section{Sampling}

Quantitative data were selected using a structure questionnaire, in a multi stage cross sectional design. We used Bennett's cluster survey sampling formula taking an assumption of a prevalence of $50 \%$, a precision of 0.032 [16] and a margin of error of $5 \%$. The sample size was 392 respondents. On adjusting for non-response, at a rate of $10 \%$, the final sample size was 431 respondents. The study used a three-stage sampling procedure; in the first stage, we randomly selected five sub-counties out of the twenty in the district. In this study, a cluster was equivalent to a village. We randomly selected five subcounties out of the twenty-three and from each sub county, we selected two parishes to give a total of ten parishes. A list of all villages from the selected parishes was then used to randomly select the total of 56 villages. We then interviewed seven adolescents 9-15 years, eligible for the HPV vaccine from each village using the Village Health Team's (VHT) guide, and taking only those who were residents of the selected villages in Mbale district for at least 2 years. A consideration of 2 years was taken because the national rollout of the vaccine was done in 2015. Care takers and adolescents who were not found in their homes after three consecutive visits were replaced with the next household. If a care taker was too ill to take the interview, they were excluded and replaced.

Health system factors were assessed through key informant interviews and an observation checklist. We conducted six key informant interviews with the district 
health team members who had an expert opinion about the health services factors that influence uptake of HPV vaccination in the district. The district team members included the following: the District Health Officer for maternal and child health, the District Cold Chain Technician and health facility In-charges. The numbers of Key Informant Interviews were deemed sufficient when additional interviews yielded little new information on the core study objectives. The interviews were audio recorded after informed verbal consent was obtained from the participants. We observed for key vaccines, supplies in selected health facilities within the sub-counties using the World Health Organization (WHO) checklist for vaccines and supplies.

\section{Dependent and independent variables}

The dependent variable was uptake of the HPV vaccine, this was measured by having a vaccination card that indicates the number of doses attained and recall of obtaining an injection on the left upper arm if the child was between 9 and 15 years. Initiation was defined as having received at least one of the recommended two dose series of the HPV vaccine and Uptake was defined as completing the two doses of the HPV vaccine.

\section{Data analysis}

Quantitative data were entered into Excel 2010, and then exported to Stata Version 13 for statistical analysis. The data were97 summarized into frequencies and proportions for categorical variables and mean. At bivariate level of analysis, Prevalence Ratio (PR) measure was used to assess relationship between the dependent variable (HPV vaccine uptake) and the independent factors. The prevalence ratios were computed using a generalized linear model with Poisson family and a log link with robust errors. At multivariable analysis, all the independent factors with a $P$ value less than 0.15 at bivariate analysis were included in the multivariable model to obtain the adjusted Prevalence ratios. The backward elimination approach was used to obtain the best model with the log likelihood that was closer to zero. The significance level for all the analysis was set at $P \leq 0.05$. The model comprised of age group, tribe, religion, and occupation, having many options from which to receive the HPV vaccine, knowing where to report side effects, having received any other vaccines, getting HPV vaccine together with other services, knowing where to report the side effects, and receiving adequate information about the vaccine.

For qualitative data, audio tape recordings were all together transcribed verbatim, coded and uploaded qualitative data analysis software MAXQDA version 12 . Recurring themes were identified within and between each interview [17]. Two independent researchers were involved in coding. These transcripts were scrutinized to ensure reliability in the use of codes between the coders. The independent lists of codes were reviewed to assess inter-coder agreement. Discrepancies were clarified and resolved by comparing each coder's results with raw data until consensus was reached. A list of codes was then finalized. The codes were based on the study objectives.

Data was then condensed through expressive, textbased summaries and data display matrices. The matrices facilitated to distinguish among the themes and groups. Quotes were then selected that were representative of the main themes.

\section{Results}

A total of 407 respondents were interviewed from the calculated sample size of 432 giving a non-response rate of $9 \%$.

\section{Socio-demographic characteristics of the respondents}

Table 1 shows the baseline characteristics of the respondents; the mean age was $11.8 \pm 1.8$ years with a minimum age of 9 years and a maximum of 15 years. $75 \%$ (306/407) of the respondents lived in rural areas. The care takers of the adolescents were mostly married (73.5\%) with half of them having attained up to primary level of education (50.1\%). Most of the respondents were of the Gishu tribe (71.3\%), and more than two thirds were of Muslim faith (41.5\%). Most (71\%) of the respondents lived approximately $1 \mathrm{~km}$ to $3 \mathrm{~km}$ from a health facility.

\section{Initiation of the HPV vaccine}

Figure 1 shows the total number of adolescents interviewed, 49\% (200/407) had initiated the vaccination, of these, adolescents that had initiated the HPV vaccine, $13.8 \%(56 / 407)$ had received both doses and thus completed the vaccination. See Fig. 1.

Table 2 shows the main reasons for not receiving the vaccine. The total number of respondents was 348 because it included only those who had either received one dose or none. Lack of awareness was the main reason given by $45 \%(182 / 348)$ of the adolescents. While some respondents mentioned that they were not aware about the HPV vaccine, some were not aware of the number of doses that they must receive and others were not aware of the schedule or interval of the vaccines. Less than $2 \%$ $(6 / 348)$ of the respondents mentioned unfriendly health workers as a major reason for failure to obtain the vaccine, while $4 \% 4 \%(14 / 348)$ of the respondents who had received one dose were aware that they were due for a second dose. Other reasons for not vaccinating include reluctance to vaccinate, being afraid of vaccines, and myths about the vaccines. 
Table 1 Shows the socio demographic characteristics of the participants

\begin{tabular}{|c|c|c|}
\hline Characteristic & Frequency $(N=407)$ & Percentage (\%) \\
\hline \multicolumn{3}{|l|}{ Age (years) } \\
\hline $9-10$ & 116 & 28.5 \\
\hline $11-12$ & 130 & 31.9 \\
\hline $13+$ & 161 & 39.6 \\
\hline \multicolumn{3}{|l|}{ Residence } \\
\hline Urban & 101 & 24.8 \\
\hline Rural & 306 & 75.2 \\
\hline \multicolumn{3}{|l|}{ Marital status of caretaker } \\
\hline Single & 18 & 4.5 \\
\hline Married & 299 & 73.5 \\
\hline Divorced/separated & 49 & 12.0 \\
\hline Widowed & 41 & 10.0 \\
\hline \multicolumn{3}{|c|}{ Education level of caretaker } \\
\hline No formal education & 46 & 11.3 \\
\hline Primary level & 204 & 50.1 \\
\hline Secondary level & 138 & 33.9 \\
\hline Tertiary level & 19 & 4.7 \\
\hline \multicolumn{3}{|l|}{ Religion } \\
\hline Catholic & 75 & 18.4 \\
\hline Anglican & 129 & 31.7 \\
\hline Moslem & 169 & 41.5 \\
\hline Others & 33 & 8.4 \\
\hline \multicolumn{3}{|l|}{ Tribe } \\
\hline Bagishu & 290 & 71.3 \\
\hline Banyole & 62 & 15.2 \\
\hline Others & 55 & 13.5 \\
\hline \multicolumn{3}{|l|}{ Occupation of care taker } \\
\hline House wife & 59 & 14.5 \\
\hline Formal employment & 22 & 5.4 \\
\hline Business women & 131 & 32.2 \\
\hline Farmer & 195 & 47.9 \\
\hline \multicolumn{3}{|l|}{ Number of siblings } \\
\hline One to two & 61 & 15.0 \\
\hline Three to four & 192 & 47.2 \\
\hline Five and more & 154 & 37.8 \\
\hline \multicolumn{3}{|l|}{ Distance to health facility } \\
\hline Less than 1 km & 78 & 19.2 \\
\hline Between 1 and $3 \mathrm{~km}$ & 289 & 71.0 \\
\hline Greater than $3 \mathrm{~km}$ & 40 & 9.8 \\
\hline
\end{tabular}

Factors associated with uptake of the HPV vaccine

Table 3 shows that the prevalence of uptake was two times higher among the age group of 11 to 12 years (PR 2.1, 95\% CI 1.0-4.4) compared to those who are 9 to 10 years. It was also twice higher among the Banyole ethnic group (UPR2.2, 95\% CI, 1.18-4.04) compared to the Bagishu, it was also six times higher among adolescents whose care takers were business women (unadjusted PRR 5.9, 95\% CI 2.0-16.9) compared to those who were housewives.

Uptake for the vaccine was also twice higher among those who had received other childhood vaccines (UPR 1.8, 95\% CI 1.05-3.01), and seven times higher among those who obtained HPV vaccine from outreach clinics (UPR 7.4, 95\% CI 3.6-15.15). Additionally, uptake was eleven times higher among those who received an explanation on the side effects of the HPV vaccine (UPR 10.6, 95\% CI 5.5-20.57), six times higher among those who got the vaccines alongside other services (unadjusted PRR 5.8, 95\%CI 3.4-9.7), seven times higher among adolescents who had many options from where to receive $\mathrm{HPV}$ vaccine (unadjusted PRR 7.1, 95\% CI 3.5-14.18) and three times higher among those with knowledge of where to report side effects (UPR $3.095 \%$ CI 1.7-5.1).

Table 4; shows the multivariable analysis, after adjusting for possible confounders, the prevalence of uptake of the HPV vaccine was two and a half times higher among girls who had received the vaccine from an outreach clinic APR 2.6,95\% CI: 1.2-5.9) compared to those who obtained from static sites. It was also three times higher among those who received an explanation for possible side effects (APR 2.7, 95\% CI 1.1-6.4) compared to those who didn't get an explanation. Prevalence was also twice higher among adolescents who received vaccines together with other services (APR 2.3, 95\% CI 1.1-4.6) and four times more among adolescents who had many options from where to receive the HPV vaccine (APR 3.6, 95\% CI 1.6-8.1) after controlling for all the other significant variables at bivariate analysis. See table below.

\section{Barriers to service delivery}

In the study, the major barriers to service delivery from the key informant interviews included low financing, myths about the vaccine, unclear communication on the target for the vaccine's coverage and transport challenges to reach the adolescents in the community. Funding for immunization activities was previously provided by other organizations that supplemented the Primary Health Care (PHC) funds but this was not happening at the time of the study. This is affirmed by one key informant who states that;

"Previously, GAVI was supplementing the PHC Funds but in the last financial year, it has been hard to manage and I am sure that some facilities have not been able to conduct outreaches in both the 


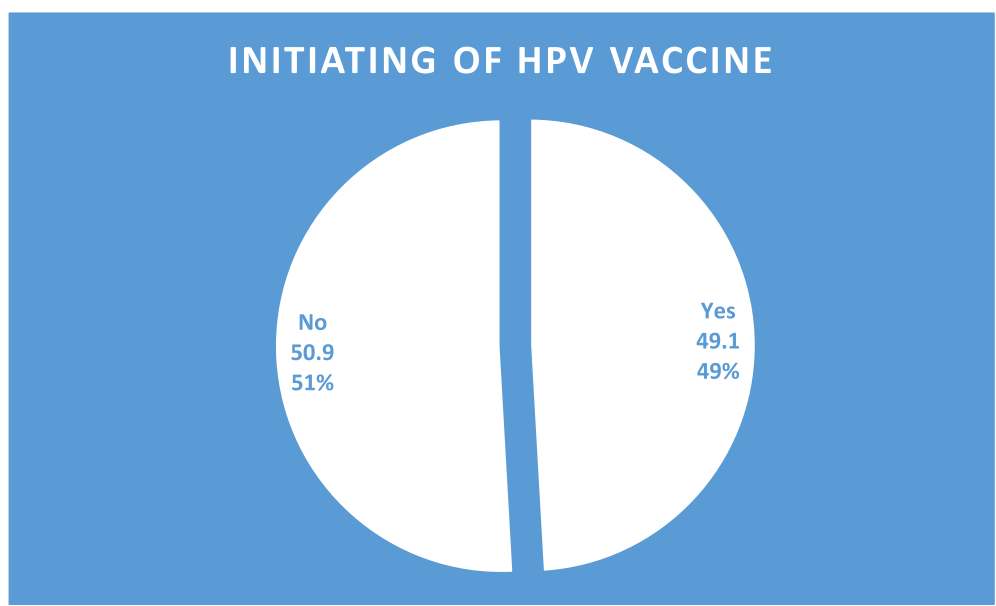

Fig. 1 Bar chart showing initiation of the HPV vaccine among adolescent girls

schools and the community" (Key informant 2, DHT).

Some key informants revealed that private schools and private health facilities are not given the HPV vaccine and this creates inequity in access for those who prefer to utilize private health facilities for receipt of the vaccine and girls in private schools.

"We supply the vaccine to the public and private not for profit health facilities, we are not giving the private clinics, this is because many of them are not equipped with the cold chain and they do not report to us."

(Key Informant 1, DHT)

"We give out this vaccine to government schools only, the private schools don't benefit because they have to obtain parental consent for their pupils to get it. In

Table 2 Major reasons for failing to obtain (initiate and or complete) the HPV vaccine

\begin{tabular}{lll}
\hline Reason & Frequency $(N=348)$ & Percentage \\
\hline Distance to health facility & 50 & 14.4 \\
No money & 35 & 10.0 \\
Unfriendly health workers & 6 & 1.7 \\
Lack of awareness & 182 & 52.3 \\
Not due for second dose & 14 & 4.0 \\
Others & 61 & 17.5 \\
Total & $348^{*}$ & 100 \\
\hline
\end{tabular}

*Total is more than the number of those who have not got the HPV vaccine since even those who had got one dose were asked the question the Government schools, the school authority gives the consent".

(Key Informant 3 health facility in-charge)

\section{Facilitators to service delivery}

HPV vaccine delivery has been made easier through the school-based delivery approach because the target group was clear but there was confusion as to whether to vaccinate those in primary five since the target class is primary four. With the school-based approach, health facilities have been able to liaise with schools to make it easy for the adolescents to receive HPV vaccine as stated by one key informant below.

"Health facilities liaise with the schools so that arrangements are made for the HPV vaccination, for example they set aside a classroom where the equipment can be placed so that the vaccination can take place".

(Key Informant 2, Healthy facility in-charge)

\section{Barriers for human resources for health}

The major barriers to human resources for health mentioned were the inadequate staff to run the work in the health center and insufficient training on HPV vaccine.

"We have few staff, which also compromises our service delivery. If some health workers go to the outreach clinic, you can feel the impact in the health facility when a few of us are left here".

(Key Informant 1, Healthy facility in-charge)

Despite the inadequate staff at the health facility, the VHTs and other community mobilisers support the health workers in mobilizing the community to take their daughters for vaccination thus motivating them. In 
Table 3 Bivariate analysis of independent factors associated with HPV vaccine uptake among adolescents

\begin{tabular}{|c|c|c|c|c|}
\hline \multirow[t]{2}{*}{ Variables } & \multicolumn{2}{|c|}{ Received two doses of } & \multirow{2}{*}{$\begin{array}{l}\text { UPR (95\% } \\
\text { Confidence } \\
\text { Interval) }\end{array}$} & \multirow[t]{2}{*}{$P$-value } \\
\hline & HPV vaccine & & & \\
\hline Education level of caretaker & Yes (\%) & No (\%) & & \\
\hline None & $6(10.7)$ & $40(11.4)$ & 1.0 & \\
\hline Primary & 18(32.1) & $186(53)$ & $0.7(0.27-1.70)$ & 0.407 \\
\hline Secondary \& above & $32(57.1)$ & 125(35.6) & $1.6(0.65-3.73)$ & 0.316 \\
\hline \multicolumn{5}{|l|}{ Age group } \\
\hline $9-10$ & $9(16.1)$ & 107(30.5) & 1.0 & \\
\hline $11-12$ & 33(58.9) & 165(47.0) & $2.1(1.0-4.4)$ & $0.042^{*}$ \\
\hline $13-15$ & 14(250.) & $79(22.5)$ & $1.9(0.84-4.4)$ & 0.121 \\
\hline \multicolumn{5}{|l|}{ Religion } \\
\hline Catholics & 13(23.2) & $62(17.7)$ & 1.0 & \\
\hline Anglicans & $24(42.86)$ & 105(29.9) & $1.1(0.55-2.10)$ & 0.837 \\
\hline Muslims & 14(25.0) & 155(44.2) & $0.5(0.22-1.01)$ & 0.055 \\
\hline Others & $5(8.9)$ & $29(8.3)$ & $0.9(0.30-2.38)$ & 0.755 \\
\hline \multicolumn{5}{|l|}{ Currently in school } \\
\hline Yes & $52(92.9)$ & $323(92.0)$ & $1.1(0.4-3.04)$ & 0.842 \\
\hline No & $4(7.1)$ & 28(7.98) & 1.0 & \\
\hline \multicolumn{5}{|l|}{ Nature of the school } \\
\hline Government aided & 15(28.3) & $240(73.4)$ & 1.0 & \\
\hline Private funded & $38(71.7)$ & $87(26.6)$ & $1.1(0.59-.955)$ & 0.811 \\
\hline \multicolumn{5}{|l|}{ Tribe } \\
\hline Bagishu & $32(57.1)$ & 258(73.5) & 1.0 & \\
\hline Banyole & $15(27)$ & $47(13.4)$ & $2.2(1.18-4.04)$ & $0.012^{*}$ \\
\hline Others & $9(16.1)$ & $46(13.1)$ & $1.5(0.707-3.10)$ & 0.296 \\
\hline \multicolumn{5}{|l|}{ Occupation } \\
\hline Housewife & $5(8.9)$ & $54(15.4)$ & 1.0 & \\
\hline Business woman & 11(19.6) & $11(3.1)$ & $5.9(2.049-16.9)$ & $<0.001^{* *}$ \\
\hline Formal employment & $15(26.8)$ & 116(33.1) & $1.4(0.49-3.72)$ & 0.56 \\
\hline Farmer & 25(44.6) & 170(48.4) & $1.5(0.579-3.95)$ & 0.398 \\
\hline \multicolumn{5}{|l|}{ Distance to the health facility } \\
\hline Less than $1 \mathrm{~km}$ & $17(30.4)$ & $61(17.4)$ & 1.0 & \\
\hline $1 \mathrm{~km}-3 \mathrm{~km}$ & $34(60.7)$ & 255(72.7) & $1.9(0.30-0.97)$ & $0.038^{*}$ \\
\hline More than $3 \mathrm{~km}$ & $5(8.9)$ & $35(9.8)$ & $1.8(0.21-1.6)$ & 0.274 \\
\hline \multicolumn{5}{|c|}{ Obtained HPV vaccine from an outreach clinic } \\
\hline Yes & $47(83.9)$ & $121(34.5)$ & 7.4(3.6-15.2) & $<0.001^{* *}$ \\
\hline No & $9(16.1)$ & 230(65.5) & 1.0 & \\
\hline \multicolumn{5}{|c|}{ Received an explanation on side effects of vaccine } \\
\hline Yes & $45(80.4)$ & $68(19.4)$ & 10.6(5.5-20.6) & $<0.001^{* *}$ \\
\hline No & 11(19.6) & 283(80.6) & 1.0 & \\
\hline \multicolumn{5}{|l|}{ Paid for HPV vaccine? } \\
\hline Yes & $4(7.1)$ & $11(3.1)$ & $2.0(0.72-5.5)$ & 0.178 \\
\hline No & $52(92.9)$ & 340(96.9) & 1.0 & \\
\hline \multicolumn{5}{|c|}{ Had many options from where to receive HPV vaccine } \\
\hline Yes & $44(81.5)$ & 107(31.3) & $7.1(3.5-14.2)$ & $<0.001^{* *}$ \\
\hline
\end{tabular}


Table 3 Bivariate analysis of independent factors associated with HPV vaccine uptake among adolescents (Continued)

\begin{tabular}{|c|c|c|c|c|}
\hline \multirow[t]{2}{*}{ Variables } & \multicolumn{2}{|c|}{ Received two doses of } & \multirow{2}{*}{$\begin{array}{l}\text { UPR (95\% } \\
\text { Confidence } \\
\text { Interval) }\end{array}$} & \multirow[t]{2}{*}{$P$-value } \\
\hline & \multicolumn{2}{|c|}{ HPV vaccine } & & \\
\hline No & $10(18.5)$ & 235(68.7) & 1.0 & \\
\hline \multicolumn{5}{|c|}{ Received any other childhood vaccines } \\
\hline Yes & $28(50)$ & 118(33.6) & $1.8(1.05-3.0)$ & \multirow[t]{2}{*}{$0.030^{*}$} \\
\hline No & $28(50)$ & $233(66.4)$ & 1.0 & \\
\hline \multicolumn{5}{|c|}{ Received HPV vaccine alongside other services } \\
\hline Yes & $26(46.4)$ & $27(7.7)$ & $5.8(3.4-9.7)$ & \multirow[t]{2}{*}{$<0.001^{* *}$} \\
\hline No & $30(53.6)$ & $323(92.3)$ & 1.0 & \\
\hline \multicolumn{5}{|c|}{ Received adequate information about the vaccine } \\
\hline Yes & $35(62.5)$ & $53(15.1)$ & $6.0(3.5-10.4)$ & \multirow[t]{2}{*}{$<0.001^{* *}$} \\
\hline No & $21(37.5)$ & 298(84.9) & 1.0 & \\
\hline \multicolumn{5}{|c|}{ Heard of someone with side effects of HPV vaccine } \\
\hline Yes & $5(8.9)$ & $9(2.6)$ & $2.8(1.0-6.8)$ & \multirow[t]{2}{*}{$0.031^{*}$} \\
\hline No & $51(91.1)$ & $342(97.4)$ & 1.0 & \\
\hline \multicolumn{5}{|c|}{ A of where to report side effects of HPV vaccine? } \\
\hline Yes & 20(35.7) & $44(12.5)$ & $3.0(1.7-5.1)$ & \multirow[t]{2}{*}{$<0.001^{* *}$} \\
\hline No & $36(64.3)$ & $307(87.5)$ & 1.0 & \\
\hline
\end{tabular}

Cl Confidence interval, Significance level $P<0.05,{ }^{*} P<0.01,{ }^{*} P<0.001$

addition, health workers were motivated to work with the available Primary Health Care funds. The team work and role played by the Village health teams and other mobilisers in the community motivated them.

"PHC funds have helped to facilitate vaccinators and this is a good strategy for us. In addition, we use phone messages to thank them for the good work they do despite the hardship" (Key informant 1, District Health Team).

\section{Barriers to vaccines, supplies and medicines}

Inconsistency in vaccine supply was noted in both the checklists and from various key informants and records in health facilities; the first supply of vaccines doses was underestimated.

"The inconsistence in vaccine supply is a major barrier to completion of the doses, and it is something that I know is beyond the District Health Office to handle."

(Key informant 1, DHT)

"Supply of the vaccine is very poor and inconsistent. Despite this, we give out the doses as and when we receive the stock, but in that case, we can't ascertain the efficacy of the vaccine".
(Key informant 2, DHT)

The integration of the HPV vaccines with other services such as child days plus helps to increase coverage by taking advantage of the existing infrastructure to provide the vaccine. This is expressed by some key informants.

"Furthermore, this is an integrated service and people get very many services at once, may be this has contributed to the success".

(Key informant 4, Health facility in-charge)

\section{Discussion}

The study estimated the level of uptake for the bivalent HPV vaccine in Mbale district, in eastern Uganda and found that $14 \%$ of the study participants were fully vaccinated. However, the estimated uptake in this survey was lower than what the district reports for HPV uptake of $32 \%$. This discrepancy may be due to errors in reporting from various health facilities into the Health Management Information System [18, 19]. The variance may also be due to unreliable census figures, an unclear denominator due to the stringent eligibility criteria for adolescent girls who should receive the HPV vaccine, the uptake was similar to a study done in Lira district, Northern Uganda which found that $14 \%$ of the adolescents were fully vaccinated [20].

The low uptake of the HPV vaccine was attributed to inadequate training among health workers about the vaccines; Uganda merged the measles campaign, Polio 
Table 4 Multivariable analysis of independent factors associated with HPV vaccine uptake among adolescents

\begin{tabular}{|c|c|c|c|c|}
\hline Variables & $N=407(\%)$ & UPR (95\% Cl) & Adj.PR(95\%Cl) & $P$-value \\
\hline \multicolumn{5}{|c|}{ Age group } \\
\hline $9-10$ & $116(28.5)$ & 1.0 & 1.0 & \\
\hline $11-12$ & 198(48.6) & $2.1(1.0-4.4)$ & $1.3(0.57-3.01)$ & 0.527 \\
\hline $13-15$ & $93(22.9)$ & $1.9(0.84-4.4)$ & $1.2(0.48-3.27)$ & 0.64 \\
\hline \multicolumn{5}{|c|}{ Obtaining vaccine from outreach clinic } \\
\hline Yes & 168(41.3) & 7.4(3.6-15.15) & $2.6(1.16-5.86)$ & $0.020^{*}$ \\
\hline No & 239(58.7) & 1.0 & 1.0 & \\
\hline \multicolumn{5}{|c|}{ Explanation on side effects of HPV vaccine } \\
\hline Yes & $113(27.8)$ & 10.6(5.5-20.57) & $2.7(1.13-6.4)$ & $0.024^{*}$ \\
\hline No & 294(72.2) & 1.0 & 1.0 & \\
\hline \multicolumn{5}{|c|}{ Many options to receive vaccine } \\
\hline Yes & $151(38.1)$ & 7.1(3.5-14.18) & $3.6(1.58-8.13)$ & $0.002^{*}$ \\
\hline No & 245(61.9) & 1.0 & 1.0 & \\
\hline \multicolumn{5}{|c|}{ Received other vaccines } \\
\hline Yes & 146(35.9) & $1.8(1.05-3.01)$ & $0.6(0.317-1.2)$ & 0.206 \\
\hline No & $261(64.1)$ & 1.0 & 1.0 & \\
\hline \multicolumn{5}{|c|}{ Got vaccine with other services } \\
\hline Yes & $53(13.0)$ & $5.8(3.4-9.7)$ & $2.3(1.11-4.59)$ & $0.024^{*}$ \\
\hline No & $353(86.7)$ & 1.0 & 1.0 & \\
\hline \multicolumn{5}{|c|}{ Received adequate information about HPV vaccine } \\
\hline Yes & 88 & $6.0(3.5-10.4)$ & $1.6(0.85-3.33)$ & 0.137 \\
\hline No & 319 & 1.0 & 1.0 & \\
\hline \multicolumn{5}{|c|}{ Heard of someone with side effects of vaccine } \\
\hline Yes & 14 & $2.8(1.0-6.8)$ & $1.2(0.38-3.9)$ & 0.723 \\
\hline No & 393 & 1.0 & 1.0 & \\
\hline
\end{tabular}

Supplementary Immunization Activities (SIA) and HPV vaccine introductory activities due to limited bandwidth within the Uganda National Expanded Program on Immunization (UNEPI) and insufficient funds to cover all activities. However, this led to key critical shortfalls in HPV implementation: training of health workers on HPV vaccine was reduced from 3 days to 1 day; and there was no social mobilization messaging on HPV vaccine because the vaccine had not yet arrived in the country, hence demand couldn't be increased yet the vaccine was not immediately available.

Additionally, low uptake may also be attributed to the lack of Information, Education and Communication (IEC) materials on HPV vaccine in health facilities, schools and other communal places such as markets. These IEC materials are usually a way of communicating health related information to a vast majority of the population, this is in agreement with findings from one study which stressed the lack of education material on HPV vaccination given by health professionals to young adolescents as a barrier to vaccine uptake and emphasized the need to improve education about cervical cancer, prevention and HPV vaccination [21]. Several studies have highlighted the need for health workers to be trained to provide adequate information about this vaccine. In many of these studies, health workers are the most preferred source of information and influence the decision to vaccinate [22-24]. Furthermore, receiving adequate information from a health care provider greatly improved uptake of the vaccine, this finding is similar to other studies, where healthcare professionals impacted the choice for adolescents to receive the HPV vaccine $[25,26]$ and these decisions were shaped by confidence in the vaccination program and healthcare providers. This may call for health workers to provide a brief discussion on the vaccine, its benefits and possible side effects prior to administration [25].

\section{Brief explanation about the vaccine}

An interventional study conducted in the United States of America showed the effect of a brief (10 minutes) group HPV educational session on knowledge and intent to vaccinate among young adults. Individuals in the intervention group were three times more likely to take on the vaccine $[27,28]$; this is similar to findings from this study where girls who received an explanation on the side effects of the vaccine were almost three times more likely to take on the vaccine as compared to those who didn't receive an explanation. Findings from this study show that adolescents who received adequate information about HPV vaccine were more likely to receive it, and this is similar to findings from another study conducted in Kenya which discovered that perceiving oneself to be adequately informed was a strong determinant of HPV vaccine uptake [29]. This means that health workers need to be trained to provide the necessary knowledge on HPV vaccine prior to provision on the vaccine to the adolescent girls.

\section{Low awareness among the target beneficiaries and caretakers}

Lack of awareness was another major factor influencing initiation and uptake of the HPV vaccine, this finding is similar to a study which sought to understand suboptimal HPV vaccine uptake among ethnic minority adolescents, with the strongest predictor of initiation reported as vaccine awareness [30, 31]. The study also highlighted that the lack of information about HPV vaccine and where to obtain it by mothers negatively influenced their decision making [30]. Additionally, a study among women in Malawi, showed that respondents believed that HPV vaccine uptake would be increased if information were dispersed throughout the community, since they strongly believed that this would address the 
challenge of low awareness on HPV vaccine [32]. This shows the importance of social mobilization especially for new vaccines that are outside of the known target age group.

\section{Inadequate human resources for health}

This study also found that human resources for health were inadequate in various health facilities to provide HPV vaccine. This may be due to the inadequate staffing levels at the district which is estimated at $73 \%$ at the time of the study. Health workers usually have to leave the health facility and move to schools to provide the HPV vaccine to the girls; this leaves the work at the health facility to a few health workers and increases the burden on the few staff members who remain at the facility. This finding is similar to one study that looked at uptake of HPV vaccine in low and middle income countries and also revealed that human resources for health were inadequate for HPV vaccine delivery [33-35]. The implication of this is that the Ministry of Health will need to find more innovative ways of increasing the human resource needed to provide the vaccine to this special age group. The insufficient human resources was reported as a challenge to vaccine delivery, this is consistent with findings from another study where human resources find it a challenge to go to outreach clinics, they use "vaccinators" to help ease on the work load [33].

\section{Vaccine and supplies}

The availability of HPV vaccines was mentioned throughout interviews as having influence over adolescent girl's uptake of HPV vaccine. As noted among the key informants, many times, the adolescents found the vaccine out of stock, and this worried the health workers about the efficacy of the vaccine since the second dose was received much later than the recommended time of 6 months interval. This finding is similar to a study in Malawi that elaborated the vaccine stock outs and inconsistency in supply as barriers to uptake [32].

The inconsistency in supply may be due to the fact that new vaccines impose pressure on the health systems of most developing countries. As a result, they are faced with challenges in their vaccine supply and logistics systems [36]. Additionally, storage capacity bottlenecks can occur at national, regional, and district levels and system inefficiencies threaten vaccine access, availability, and quality. At the national level, HPV forecasts and supplies were adjusted to cater for peak demand during the months of April and October while at district level, due to the limited knowledge of target age group, health facilities forecasts and deliveries did not align with monthly need including peak periods resulting in surplus in supply in some health facilities and stock-outs in others.

As Uganda adopts the HPV vaccine, the health system must attempt to reach people at different ages and in new settings, as a result, the logistics systems must be strengthened and improved.

\section{Service delivery}

Integration of services was found to significantly increase uptake of the HPV vaccine. This is because adolescents get extra services such as deworming, family planning, HIV testing and health education that are given at these outreach clinics. Gavi recommends that integrated programs offer opportunities for other age-relevant services such as de-worming and nutritional supplements. This integrated approach presents an opportunity to reduce the cost and burden on health systems of delivering separate interventions [37].

This study found the cold chain (fridges, thermometer, vaccine carriers, and storage space) and the infrastructure was adequate, this is contrary to findings from two previous studies, where infrastructure for the delivery of the HPV vaccine was found to be lacking $[33,38]$. The cold chain may have been found to be better due to the continuous support of the United Nations children's fund (UNICEF) and Gavi support to the Expanded Program on Immunization in the district. There has also been an improvement in infrastructure because of continuous and more preparation for cold chain due to the introduction of Pneumococcal Conjugate Vaccine (PCV), and the change from Oral Polio Vaccine (OPV) to Injectable Polio Vaccine (IPV) plus other planned new vaccine introductions.

The similarly striking low rates of HPV vaccination in a study among Cambodian American teenagers highlighted the need to improve vaccination outreach [39];. These findings are similar to this study and thus can be used to develop targeted public health HPV vaccination programs for various geographical groups, which will reduce cervical cancer disparities. Outreach clinics are suitable, particularly for children out of school.

This study revealed that cost was not a barrier to obtaining the vaccine, this is contrary to another study where cost was a barrier to vaccination [40]. The difference in the findings may be due to the fact that the HPV vaccine is provided free of charge by the Government of Uganda and subsidized by the Global Alliance for Vaccines and Immunization [41]. One more study conducted on predictors of HPV vaccination among daughters of low-income Latina mothers identified independent predictors of HPV vaccine uptake, and low worry about how to pay for the vaccine was a predictor for vaccination [42]. 


\section{Study limitations and strengths}

This study was cross-sectional in nature and therefore we cannot infer a causal relationship between awareness of the vaccine and subsequent uptake. This means that this study cannot depict that awareness on the HPV Vaccine always results in uptake. Nonetheless, the study addressed one of the key areas which is salient in the effort needed to enhance HPV Vaccine uptake in the country.

\section{Conclusion}

Uptake for the HPV vaccine in this study was defined as completing two doses of the vaccine. In this study, uptake was $14 \%$. This is much lower than the $80 \%$ national HPV vaccine coverage target. Lack of awareness about HPV vaccine was found to be the main reason for the low uptake of the HPV vaccine. Besides, lack of communication and advocacy on the vaccine to raise awareness also hampered its uptake.

Factors that positively influenced uptake of the vaccine include receiving an explanation for possible HPV vaccine side effects, having many options from where to get the vaccine, getting the vaccine from an outreach clinic, and getting the vaccine alongside other services.

\section{Recommendations}

The Ministry of Health and implementing partners must aim at raising awareness about the HPV vaccine as a primary preventive mechanism against cervical cancer through various forms of media. In addition, the Government must nurture a public private partnership to include private health facilities in providing the HPV vaccine so as to increase coverage in areas that are mainly served by the private health services.

The District Health Team should conduct continuous on job training of health workers on HPV vaccine so that they can deliver quality information to the adolescents prior to receipt of the vaccine.

The Ministry of Health must ensure that the expected target for coverage is communicated to all relevant stakeholders so that they work towards it and are capable of monitoring and evaluating their work.

\section{Implications}

Awareness can be improved by engaging various media options for the particular age group, communicating with the care takers of the children to allay fears that may lead to hesitancy and wide distribution of the vaccine to reach as many adolescents as possible.

\section{Abbreviations}

APR: Adjusted Prevalence Ratio; DHO: District Health Officer; DHT: District Health Team; GAVI: Global Alliance for Vaccines and Immunization; HPV: Human Papilloma Virus; IEC: Information, Education and Communication; PHC: Primary Health Care; PR: Prevalence Ratio;
SIA: Supplementary Immunization Activities; UNEPI: Uganda National Expanded Program on Immunization; UPR: Unadjusted Prevalence Ratio; VHT: Village Health Team; WHO: World Health Organization

\section{Acknowledgements \\ We wish to acknowledge our Research assistants in Mbale District, Abdul Aziz Wambewo, Sibi Samuel, Abdullah Foozi and Madoi Hashim. The entire District Health team for the technical support. Dr. John Baptist Waniaye and Dr. Jonathan Wangisi. Special thanks to the administrative team at Makerere University School of Public Health and Mbale District Health Office. \\ This study was presented to the 9th conference of the Consortium of Universities for Global Health in New York City in March 2018. https://www.sciencedirect.com/science/article/pii/S2214109X18301372?via\%3 Dihub}

\section{Authors' contributions}

JN participated in designing the study, data collection, and statistical analyses and served as the lead author of the manuscript. CK and GK participated in study conception FM and NR participated in the analysis and interpretation of results. GK participated in qualitative data analysis. OAL and JNB played active roles as supervisors during all stages of the study as well as manuscript writing. All authors read and approved the final manuscript.

\section{Funding}

This study was partly funded by the Makerere University School of Public Health, through Supporting Policy Engagements for Evidence-based Decisions (SPEED) for Universal Health Coverage Project, and The German Academic Exchange Services, Deutscher Akademischer Austauschdient (DAAD). The funders provided financial support through the study that catered for all expenses incurred during the research period.

\section{Availability of data and materials}

The dataset used and analyzed during this study is available from the corresponding author upon reasonable request.

\section{Ethics approval and consent to participate}

Ethical approval to conduct this study was granted by the Higher Degrees Research and Ethics Committee (HDREC) of the Makerere University School of Public Health and the Uganda National Council of Science and Technology (UNCST). Permission was also obtained from the District Health Officer (DHO). Data was anonymized for analysis. Participants in the crosssectional quantitative and qualitative interviews provided written informed consent and assent prior to the interview. Parents and guardians of the minors provided verbal consent prior to the interview of the adolescent girl.

\section{Consent for publication}

Not applicable.

\section{Competing interests}

The authors declare that they have no competing interest.

\section{Author details}

${ }^{1}$ Department of Health Policy, Planning and Management Makerere University School of Public Health College of Health Sciences, P.O. Box 7072, Kampala, Uganda. ${ }^{2}$ Department of Disease control and Environmental Health, University School of Public Health College of Health Sciences, Kampala, Uganda. ${ }^{3}$ Department of Biomedical sciences, Makerere University, School of medicine College of Health Sciences, Kampala, Uganda. ${ }^{4}$ Department of Epidemiology and Biostatistics, School of Public Health, Makerere University, Kampala, Uganda.

Received: 13 June 2019 Accepted: 29 January 2020

Published online: 04 February 2020

\section{References}

1. International Agency for Research on Cancer. 2008

2. WHO. Human papillomavirus infection and cervical cancer WHO; 2010.

3. Anorlu RI. Cervical cancer: the sub-Saharan African perspective. Reprod Health Matters. 2008;16(32):41-9. 
4. Mutyaba T, Mirembe F, Sandin S, Weiderpass E. Male partner involvement in reducing loss to follow-up after cervical cancer screening in Uganda. Int J Gynecol Obstet. 2009;107(2):103-6.

5. WHO. Prevention of cervical cancer through screening using visual inspection with acetic acid (VIA) and treatment with cryotherapy. A demonstration project in six African countries: Malawi, Madagascar, Nigeria, Uganda, the united republic of Tanzania and Zambia. Geneva: World Health Organization. : African Population and Health Research Center, International Agency for Research on Cancer; 2012.

6. Parkin DM, Nambooze S, Wabwire-Mangen F, Wabinga HR. Changing cancer incidence in Kampala, Uganda, 1991-2006. Int J Cancer. 2010;126(5): 1187-95.

7. Parkin DM, Almonte M, Bruni L, Clifford G, Curado M-P, Pineros M. Burden and trends of type-specific human papillomavirus infections and related diseases in the Latin America and Caribbean region. Vaccine. 2008;26:L1L15.

8. Banura C, Sandin S, van Doorn L-J, Quint W, Kleter B, Wabwire-Mangen F, et al. Research article type-specific incidence, clearance and predictors of cervical human papillomavirus infections (HPV) among young women: a prospective study in Uganda; 2010.

9. PATH. Protecting girls and women from cervical cancer. 2008

10. Boyce T, Holmes A. Addressing health inequalities in the delivery of the human papillomavirus vaccination programme: examining the role of the school nurse. PLoS One. 2012;7(9):e43416.

11. WHO. Human papillomavirus vaccines, WHO position paper. Wkly Epidemiol Rec. 2009;84:118-31 WHO; 2009.

12. Grace JA. Adolescent immunization: challenges and opportunities. J Sch Nurs. 2006;22(2):87-93

13. Kharbanda EO, Kahn JA. Vaccinating teens: current guidelines, challenges, and opportunities. Pediatr Ann. 2010;39(8):483-9.

14. $\mathrm{MOH}$. Uptake of HPV vaccine per district in Uganda: Ministry of Health Uganda; 2016. Kampala, Uganda

15. Raffle AE. Challenges of implementing human papillomavirus (HPV) vaccination policy. BMJ: British Med J. 2007:335(7616):375-7.

16. Turner AG. Sampling frames and master samples. United Nations secretariat statistics division; 2003.

17. Strauss A, Corbin J. Grounded theory methodology. Handbook Qualitat Res. 1994;17:273-85.

18. Kiberu VM, Matovu JK, Makumbi F, Kyozira C, Mukooyo E, Wanyenze RK. Strengthening district-based health reporting through the district health management information software system: the Ugandan experience. BMC Med Inform Decis Mak. 2014;14(1):40.

19. Garrib A, Herbst K, Dlamini L, McKenzie A, Stoops N, Govender T, et al. An evaluation of the district health information system in rural South Africa. SAMJ: S Afr Med J. 2008:98(7):549-52.

20. Kisaakye E, Namakula J, Kihembo C, Kisakye A, Nsubuga P, Babirye JN. Level and factors associated with uptake of human papillomavirus infection vaccine among female adolescents in Lira District, Uganda. Pan Afr Med J. 2018;31:184

21. La Torre G, De Vito E, Ficarra MG, Firenze A, Gregorio P, Boccia A. Is there a lack of information on HPV vaccination given by health professionals to young women? Vaccine. 2013;31(42):4710-3.

22. Kessels SJ, Marshall HS, Watson M, Braunack-Mayer AJ, Reuzel R, Tooher RL. Factors associated with HPV vaccine uptake in teenage girls: a systematic review. Vaccine. 2012;30(24):3546-56.

23. Marshall $H$, Ryan P, Roberton D, Baghurst P. A cross-sectional survey to assess community attitudes to introduction of human papillomavirus vaccine. Aust N Z J Public Health. 2007;31:235-42.

24. Mullins TL, Griffioen AM, Glynn S, Zimet GD, Rosenthal SL, Fortenberry JD, et al. Human papillomavirus vaccine communication: perspectives of 11-12 year-old girls, mothers, and clinicians. Vaccine. 2013;31(42):4894-901.

25. Ferrer HB, Trotter C, Hickman M, Audrey S. Barriers and facilitators to HPV vaccination of young women in high-income countries: a qualitative systematic review and evidence synthesis. BMC Public Health. 2014;14:700.

26. Cassidy B, Schlenk EA. Uptake of the human papillomavirus vaccine: a review of the literature and report of a quality assurance project. J Pediatr Health Care. 2012;26(2):92-101.

27. Kester LM, Shedd-Steele RB, Dotson-Roberts CA, Smith J, Zimet GD. The effects of a brief educational intervention on human papillomavirus knowledge and intention to initiate HPV vaccination in 18-26 year old young adults. Gynecol Oncol. 2014;132(Suppl 1):S9-12.
28. Dorell CG, Yankey D, Santibanez TA, Markowitz LE. Human papillomavirus vaccination series initiation and completion, 2008-2009. Pediatrics. 2011; 128(5):830-9.

29. Vermandere $H$, van Stam MA, Naanyu V, Michielsen $K$, Degomme O, Oort F. Uptake of the human papillomavirus vaccine in Kenya: testing the health belief model through pathway modeling on cohort data. Glob Health. 2016;12:72.

30. Bastani R, Glenn BA, Tsui J, Chang LC, Marchand EJ, Taylor VM, et al. Understanding suboptimal human papillomavirus vaccine uptake among ethnic minority girls. Cancer Epidemiol Biomarkers Prev. 2011;20(7):1463-72.

31. LAVMaJW JW. Mothers' attitudes towards preventing cervical cancer through human papillomavirus vaccination: a qualitative study; 2006.

32. Ports KA, Reddy DM, Rameshbabu A. Barriers and facilitators to HPV vaccination: perspectives from Malawian women. Women Health. 2013; 53(6):630-45.

33. Wigle J, Coast E, Watson-Jones D. Human papillomavirus (HPV) vaccine implementation in low and middle-income countries (LMICS): health system experiences and prospects(). Vaccine. 2013;31(37):3811-7.

34. Hongoro C, McPake B. How to bridge the gap in human resources for health. Lancet. 2004;364(9443):1451-6.

35. Kakuma R, Minas H, van Ginneken N, Dal Poz MR, Desiraju K, Morris JE, et al. Human resources for mental health care: current situation and strategies for action. Lancet. 2011:378(9803):1654-63.

36. Zaffran M, Vandelaer J, Kristensen D, Melgaard B, Yadav P, Antwi-Agyei KO, et al. The imperative for stronger vaccine supply and logistics systems. Vaccine. 2013;31:B73-80.

37. GAVI. Types of support \& FAQhttp://www.gavialliance.org/support/apply/; 2017.

38. Ladner J, Besson MH, Audureau E, Rodrigues M, Saba J. Experiences and lessons learned from 29 HPV vaccination programs implemented in 19 low and middle-income countries, 2009-2014. BMC Health Serv Res. 2016;16:575.

39. Lee $H$, Kim M, Kiang $P$, Shi L, Tan K, Chea P, et al. Factors associated with HPV vaccination among Cambodian American teenagers. Publ Health Nurs. 2016;33(6):493-501.

40. Brewer NT, Fazekas KI. Predictors of HPV vaccine acceptability: a theoryinformed, systematic review. Prev Med. 2007;45(2-3):107-14.

41. GAVI. GAVI factsheet Uganda; 2017.

42. Gerend MA, Zapata C, Reyes E. Predictors of human papillomavirus vaccination among daughters of low-income Latina mothers: the role of acculturation. J Adolesc Health. 2013;53(5):623-9.

\section{Publisher's Note}

Springer Nature remains neutral with regard to jurisdictional claims in published maps and institutional affiliations.
Ready to submit your research? Choose BMC and benefit from:
- fast, convenient online submission
- thorough peer review by experienced researchers in your field
- rapid publication on acceptance
- support for research data, including large and complex data types
- gold Open Access which fosters wider collaboration and increased citations
- maximum visibility for your research: over $100 \mathrm{M}$ website views per year
At BMC, research is always in progress.
Learn more biomedcentral.com/submissions 